\title{
TOPOLOGICAL WREATH PRODUCTS
}

\author{
A. LAKSHMI
}

(Received 1 April 1965)

The object of this note is to show that under suitable restrictions some results on the wreath product of groups can be carried over to topological groups. We prove in particular the following analogue of the well-known theorem of Krasner and Kaloujnine (see for example [2] Theorem 3.5):

THEOREM. Let $A$ and $B$ be two locally compact topological groups, and let $(C, \varepsilon)$ be an extension of $A$ by $B$. If there exists a continuous left inverse 1 of $\varepsilon$, that is to say a continuous mapping $\tau: B \rightarrow C$ such that $\tau \varepsilon$ is the identity on $B$, then there exists a continuous monomorphism of $C$ into the topological standard wreath product of $A$ by $B$.

We begin by defining ${ }^{2}$ topological wreath products. We start from a Hausdorff topological group $A$, a Hausdorff space $Y$, and a Hausdorff topological group $B$ acting continuously on $Y$. We write $y b$ for the image of $y \in Y$ under $b \in B$, so that $y\left(b b^{\prime}\right)=(y b) b^{\prime}$, and we assume that the unit element 1 of $B$ acts as the identity on $Y$. Let $A(Y)$ denote the group of continuous functions from $Y$ to $A$ under pointwise multiplication. We now assume that $A$ is locally compact; then $A(Y)$, endowed with the compact open topology, becomes a topological group.

Next we turn $B$ into a group of automorphisms of $A(Y)$ by defining the mapping

$$
\begin{aligned}
A(Y) \times B & \rightarrow A(Y), \\
(f, b) & \rightarrow f^{b},
\end{aligned}
$$

where $f^{t} \in A(Y)$ is defined by

$$
f^{b}(y)=f\left(y b^{-1}\right)
$$

for all $y \in Y$. It is easy to verify that

$$
\left(f \cdot f^{\prime}\right)^{b}=f^{b} \cdot f^{b}, f^{\left(b b^{\prime}\right)}=\left(f^{b}\right)^{b \prime}, f^{1}=f
$$

for all $f, f^{\prime} \in A(Y)$ and all $b, b^{\prime} \in B$.

1 We use algebraic rather than topological conventions, writing mappings as right operators: in a product of mappings the left-hand factor is applied first.

2 The present definition of the Topological Wreath Product which is an improvement on the authors' original definition is due to Prof. Karl H. Hofmann to whom the author's thanks are due. 
If we now further assume that

$$
(f, b) \rightarrow f^{b}
$$

is a continuous mapping, that is to say that $B$ acts on $A(Y)$, then the semidirect product $P$ of $A(Y)$ and $B$ becomes a topological group.

LEMMA. If the Hausdorff space $Y$ is locally compact, then the mapping

$$
(f, b) \rightarrow f^{b}
$$

is continuous, and thus $P$ is then a topological group.

Proof. Let $W(K, U)$ be a neighbourhood of $f^{b}$ in $A(Y)$ in the compact open topology ${ }^{3}$ Then

$$
f^{b}(K) \subseteq U, \text { that is } f\left(K b^{-1}\right) \subseteq U .
$$

As $K b^{-1}$ is compact in $Y$ and $f$ is a continuous mapping of $Y$ into $A$, we can find a neighbourhood $V_{0}$ of $K b^{-1}$ in $Y$ such that $f\left(V_{0}\right) \subseteq U$. Next, since $Y$ is assumed to be a locally compact Hausdorff space and $\bar{V}_{0}$ is a neighbourhood of the compact set $K b^{-1}$, a closed compact neighbourhood $V$ of $K b^{-1}$ can be found such that $K b^{-1} \cong V \subseteq V_{0}$. Again, since the mapping $\left(y, b^{-1}\right) \rightarrow y b^{-1}$ is continuous in both variables and $K$ is compact, there exists a neighbourhood $M$ of $b^{-1}$ in $B$ such that

$$
(K, M) \rightarrow K M \sqsubseteq V
$$

Choose a neighbourhood $N$ of $b \in B$ such that $N^{-1} \subseteq M$, and consider the neighbourhoods $W^{\prime}=W(V, U)$ of $f$ in $A(Y)$, and $N$ of $b$ in $B$, respectively. If $\left(f^{\prime}, b^{\prime}\right) \in W^{\prime} \times N$, then $b^{\prime-1} \in M$ and

Thus

$$
f^{\prime b^{\prime}}(K)=f^{\prime}\left(K b^{\prime-1}\right) \subseteq f(K M) \subseteq f(V) \subseteq U
$$

and the lemma follows.

$$
f^{\prime b^{\prime}} \in W(K, U)
$$

We shall call the semi-direct product $P$ of $A(Y)$ and $B$ the topological wreath product of $A$ and $B$, and write $P=A$ Wr $B$, the action of $B$ on $Y$ being understood. In particular we can make $Y$ coincide with $B$, so that $B$ acts on itself by right multiplication. If $B$ is locally compact, then the topological wreath product so defined is called the topological standard wreath product.

We remark that the topological standard wreath product of two locally compact topological groups is complete. This follows from Theorems 11, 12, and 13 of Chapter 7 of [1] and from the facts that a locally compact group is

- For the notation, see [1], p. 221. The $W(K, U)$ form a sub-base for the compact open topology, but it clearly suffices to deal with neighbourhoods of this form. 
complete and that the cartesian product of two complete uniform spaces is again a complete uniform space.

We are now ready to prove the theorem enunciated in the introduction.

Proof of THE THEOREM. The algebraic part of the proof will be the same as the proof of Theorem 3.5 in [2]. We have a continuous epimorphism $\varepsilon: C \rightarrow B$ and an iseomorphism (that is an isomorphism which is also a homeomorphism)

$$
\alpha: \operatorname{ker}(\varepsilon) \rightarrow A ;
$$

moreover we are given a continuous mapping $\tau: B \rightarrow C$ (not in general a homomorphism) such that $\tau \varepsilon$ is the identity on $B$. We define a mapping

by putting, for all $y \in B$,

$$
\gamma: C \rightarrow A^{B}
$$

$$
(c \gamma)(y)=\left(\left(y(c \varepsilon)^{-1}\right) \tau c(y \tau)^{-1}\right) \alpha,
$$

and first show that $c \gamma: B \rightarrow A$ is a continuous function, for every $c \in C$.

Let $y \in B$ be arbitrary, and let $N$ be a neighbourhood of $(c \gamma)(y)$ in $A$. As $\alpha$ is continuous, there is a neighbourhood $K$ of $\left(y(c \varepsilon)^{-1}\right) \tau c(y \tau)^{-1}$ in $C$ such that $K \alpha \subseteq N$. Next, since multiplication is continuous in $C$, we can find neighbourhoods $C_{0}$ of $\left(y(c \varepsilon)^{-1}\right) \tau$ and $C_{1}$ of $(y \tau)^{-1}$ such that $C_{0} c C_{1} \subseteq K$. Then, using also the continuity of $\tau$, we choose neighbourhoods $V_{0}$ and $V_{1}$ of $y$ in $B$ such that

and thus

$$
\left(V_{0} \cdot(c \varepsilon)^{-1}\right) \tau \subseteq C_{0}, \quad\left(V_{1} \tau\right)^{-1} \cong C_{1},
$$

$$
\left(V_{0} \cdot(c \varepsilon)^{-1}\right) \tau c\left(V_{1} \tau\right)^{-1} \subseteq K .
$$

Put $V=V_{0} \cap V_{1}$; then

$$
(c \gamma)(V) \subseteq\left(\left(V_{0} \cdot(c \varepsilon)^{-1}\right) \tau c\left(V_{1} \tau\right)^{-1}\right) \alpha \subseteq K \alpha \subseteq N ;
$$

hence $c \gamma$ is continuous at $y$, and as $y \in B$ is arbitrary, it follows that $c \gamma$ belongs to $A(B)$, for every $c \in C$.

Thus $\gamma$ maps $C$ into $A(B)$, and we now show that $\gamma$ is continuous. Let $W(F, U)=\{g \mid g(F) \subseteq U\}$ be a neighbourhood ${ }^{4}$ of $c \gamma$ in $A(B)$, where $F$ is compact in $B$ and $U$ is open in $A$. We have

$$
(c \gamma)(F) \subseteq U,
$$

and it follows, using the continuity of all mappings and operations involved in the definition of $(c \gamma)(y)$, that to every $y \in F$ one can choose neighbourhoods $V(y)$ of $y$ in $B$ and $N(y)$ of $c$ in $C$ such that

- See [1], p. 221; as before in the proof of the Lemma, it suffices to deal with neighbourhoods of this form. 


$$
\left(\left(V(y) \cdot(N(y) \varepsilon)^{-1}\right) \tau \cdot N(y) \cdot(V(y) \tau)^{-1}\right) \propto \subseteq U .
$$

Since $F$ is compact, it can be covered by a finite set of neighbourhoods of the form $V(y)$, say

Put

$$
F \subseteq \bigcup_{i=1, \cdots, n} V\left(y_{i}\right)
$$

$$
N^{*}=\bigcap_{i=1, \cdots, n} N\left(y_{i}\right)
$$

then $N^{*}$ is a neighbourhood of $c$ in $C$, and we have

$$
\left(\left(F \cdot\left(N^{*} \varepsilon\right)^{-1}\right) \tau \cdot N^{*} \cdot\left(F_{\tau}\right)^{-1}\right) \alpha \subseteq U
$$

or, differently put,

$$
N^{*} \gamma \subseteq W(F, U)
$$

The continuity of $\gamma: C \rightarrow A(B)$ then follows.

We now define a mapping $\mu: C \rightarrow P$ by

$$
c \mu=c \varepsilon \cdot c \gamma .
$$

Since the topology of $P$ is the product topology of those of $B$ and of $A(B)$, and as $\varepsilon: C \rightarrow B$ and $\gamma: C \rightarrow A(B)$ are continuous, $\mu$ is also continuous. It has been shown in [2], Proof of Theorem 3.5, that $\mu$ is a monomorphism. The theorem thus follows.

Remark 1. The continuity of $\tau$, which is part of the hypothesis of our theorem, is assured in the following instance:

THEOREM 3.1 of Hochschild [3] ${ }^{5}$. Let $A$ be a connected Lie group, $B$ a simply connected Lie group, $(C, \varepsilon)$ an extension of $A$ by $B$. Then there exists an analytic mapping $\tau$ of $B$ into $C$ such that $\tau \varepsilon$ is the identity mapping on $B$.

Remark 2. In our theorem, if we further assume that $A$ and $B$ are compact, then $C$ is compact, and thus $\mu$ is a homeomorphism.

For, if $\eta$ is the canonical homomorphism of $C$ onto $C / A$, the mapping $\tau \eta$ from $B$ to $C / A$ is continuous, being the product of the continuous mappings $\tau$ and $\eta$. That $\tau \eta$ is one-to-one and onto can be proved using the fact that there is an algebraic isomorphism $\beta$ from $C / A$ to $B$. Hence $C / A$ is homeomorphic to $B$ which is compact. From a well known theorem the compactness of $C$ then follows.

I wish to express my grateful thanks to Dr. V. K. Balachandran for his encouragement and to Prof. B. H. Neumann for revising the original version of the paper.

- The author is grateful to Dr. N. Ramabadhran for pointing out this theorem to her. 


\section{References}

[1] John L. Kelley, General Topology, Van Nostrand, New York, 1955.

[2] B. H. Neumann, Hanna Neumann, and Peter M. Neumann, Wreath products and varieties of groups, Math. Zeitschr. 80, 44-61 (1962).

[3] G. Hochschild, Group extensions of Lie groups, Ann. of Math. 54, 96-109 (1951).

Department of Mathematics

University of Madras 